\title{
El Cuento: experiencia de aprendizaje y enseñanza de las ciencias
}

\author{
Dulsat Ortiz, Carles ${ }^{1}$; Rodríguez Machado, Rafael Eduardo² \\ 'Universidad Isabel I, ORCID: 0000-0003-1351-8506 \\ 2Universidade da Coruña, Facultade de Ciencias da Educación, \\ ORCID: 0000-0003-2870-8396
}

\section{RESUMEN}

La experiencia que aquí se presenta, se realizó con el alumnado de la materia de Enseñanza de las Ciencias de la Naturaleza de segundo curso del grado de educación infantil. Se pidió la elaboración de un cuento que mostrara un concepto científico. De esta manera, se perseguía que este alumnado profundizara en el conocimiento del concepto escogido a la vez que se iniciara en el uso de una herramienta metodológica como es el cuento para acercarla a la enseñanza de las ciencias en su futuro profesional como maestras de educación infantil. El resultado expresado por el alumnado fue satisfactorio tanto en la utilización del recurso como en el aprendizaje de los conocimientos de conceptos científicos.

PALABRAS CLAVE: ciencias de la naturaleza, cuento, experiencia, recurso metodológico.

\section{CITA RECOMENDADA:}

Dulsat Ortiz, Carles; Rodríguez Machado, Rafael Eduardo (2020): El Cuento: experiencia de aprendizaje y enseñanza de las ciencias. En De la Torre Fernández, E. (ed.) (2020). Contextos universitarios transformadores: Boas prácticas no marco dos GID. IV Xornadas de Innovación Docente. Cufie. Universidade da Coruña. A Coruña (págs. 71-82).

DOI capítulo: https://doi.org/10.17979/spudc.9788497497756.071

DOl libro: https://doi.org/10.17979/spudc.9788497497756 


\section{ABSTRACT}

The experience presented here was carried out with the students of the subject of Teaching of Natural Sciences of the second year of the degree of early childhood education. The preparation of a fairy tale that showed a scientific concept was requested. In this way, it was pursued that this student will deepen the knowledge of the chosen concept while they began to use a methodological tool such as the story to bring it closer to teaching science in their professional future as teachers of children's education. The result expressed by the student was satisfactory both in the use of the resource and in the learning of the knowledge of scientific concepts.

KEY WORDS: natural science, tale, experience, methodological resource. 


\section{INTRODUCCIÓN}

Esta experiencia se origina en el intento de acercar las ciencias de la naturaleza al alumnado del grado de educación infantil y, a la vez, aproximarles un recurso para el día de mañana en su función docente. Este es el motivo del título de la experiencia que se presenta a continuación: aprendizaje de las ciencias al alumnado del grado y que sean capaz de enseñar ciencias con el uso de cuentos e historias que pueden ser existentes o bien diseñadas por ellas según las necesidades que el grupo con el que trabajan pueda requerir.

Acercar las ciencias al alumnado del grado de educación infantil se debe a que el $45.8 \%$ han estado más de 5 años alejados de los contenidos de las ciencias de la naturaleza. Aunque se hace necesario el uso de algunos recursos que faciliten este acercamiento sin detrimento del resto de compañeros de aula. Se propusieron experiencias con otras metodologías (Dulsat, 2019), donde la microenseñanza conseguía no sólo acercar los conceptos científicos al alumnado, sino que también se conseguía una primera puesta en práctica de sus competencias como docentes: preparar y exponer ante un grupo los contenidos científicos, en el caso de la materia que se imparte.

En esta ocasión se propone el diseño y elaboración de un cuento a partir de la elección de un concepto muy concreto relacionado con las ciencias naturales. Para ello todos los grupos seleccionaron la segunda etapa de Educación Infantil (de 3 a 5 años). Esto requiere el conocimiento y entendimiento del concepto escogido y adaptarlo a las edades propias de la educación infantil, o transposición didáctica (Chevallard, 1991).

Además de esta transposición didáctica, cabe considerar que el alumnado ha seleccionado los conceptos para plasmarlos en el cuento a partir de sus intereses, de sus conocimientos previos (Ausubel et al., 1983) sin ninguna restricción excepto que forme parte de las ciencias de la naturaleza y que no se repitan conceptos entre los distintos grupos; a esto, cabe añadir las aportaciones de Chi y Roscoe (2002) que entienden estos conocimientos previos como mecanismos cognitivos que contribuyen a entender y construir la realidad, en cierta manera 
cómo se acercan a ella. Estos autores formulan una metodología para superar estas ideas previas a la vez que ajustar las nuevas explicaciones de la realidad.

Es habitual el uso del cuento para la enseñanza del lenguaje, saber escuchar, saber leer, imaginar la historia o la enseñanza de lenguas extranjeras (Martínez, 2011), el desarrollo del ámbito cognitivo y de las funciones ejecutivas (Guevara, 2016). También, se usa para la enseñanza de las ciencias sociales como historias que relatan los acontecimientos pasados (Pérez, 2016) o bien en la educación en valores (Pardial y Sáenz-López. 2013). Es usual utilizarlo en la enseñanza de las matemáticas (Blanco y Blanco, 2009; Fernández et al., 2014), y de las ciencias de la naturaleza (García y Pérez, 2016; Mateos et al., 2015; Orellana y Espinet, 2009), y específicamente del conocimiento del medio ambiente (Espinet, 1995; García-Castejón, 2013).

Los objetivos de esta experiencia fueron: (1) indagar en la experiencia del uso del cuento en la materia de Ensino das Ciencias da Natureza del grado de educación infantil; y, (2) mostrar las consecuencias en el alumnado de la materia de Ensino das Ciencias da Natureza del grado de educación infantil en el uso del cuento como recurso didáctico.

\section{DESCRICIÓN DE LA EXPERIENCIA}

La experiencia llevada a cabo consistió en el diseño y elaboración de un cuento, lo más original posible. Para ello, se dispuso al gran grupo de 68 alumnas en 14 grupos de 405 componentes. Cabe destacar que, del total del alumnado, formaron parte de la experiencia 62 porque 4 optaron por la no presencialidad y 2 no se presentaron en todo el curso.

Se determinaron 3 fechas, separadas ente ellas por cuatro semanas, como tutorías obligatorias, aunque podían pedir cualquier otra tutoría cuando creyeran que fuera necesario. Entre la última tutoría y la presentación del cuento en la escuela de educación infantil, también las separaban cuatro semanas más, momento en el cual debían entregar el cuento definitivo.

En la presentación del curso, se explicó el funcionamiento del mismo y se determinaron las distintas actividades que se realizarían a lo largo de él, entre las que se expuso el trabajo a 
realizar durante todo el cuatrimestre: preparación, diseño y presentación de un cuento con la explicación de un concepto científico. El título de la actividad fue: A ciencia no conto. Se marcó la fecha de la primera tutoría para dos semanas después de esta presentación del curso. En esa primera tutoría, deberían presentarse todas las integrantes del grupo, e indicar en qué formato se realizaría -digital o en papel- y tener claro el concepto científico a trabajar. Para la elaboración en formato digital se propuso el programa: Storyjumper.

Los conceptos científicos escogidos se podrían enmarcar en los siguientes campos: cinco grupos en torno al medio ambiente con títulos como, "Mar de plástico", "Contaminación en el Ártico", "Agua", "Reducción de residuos", y "Contaminación global"; un segundo grupo que se centró en aspectos relativos a los seres vivos como "Heridas", "La reproducción de las plantas", "Crecimiento de las mariposas", "El cuerpo humano" y "Crecimiento de los árboles"; y, un último grupo que presentó cuestiones del planeta Tierra como, "¿Por qué Ilueve?", "Rayos y Truenos" y "Movimiento de la Tierra". Hay un último grupo que, en vez de centrarse en conceptos científicos, presentó la historia de la científica Jane Goodall.

La segunda tutoría estaba orientada a la aproximación de la historia, de los personajes y cómo el concepto científico se introducía en ella. En este momento de la experiencia se puso especial énfasis en la transposición didáctica y en la capacidad de cada grupo de adaptar el concepto al grupo de edad al cual se dirigían. Esta adaptación del concepto se refiere tanto a la imagen como al texto que la acompaña, de tal manera que fuera entendible y asimilable para las edades escogidas. Estas tutorías fueron más largas porque, primero, requería que el grupo entendiera y comprendiera el concepto científico, y, segundo, debían adaptar ese concepto al lenguaje propio de la edad seleccionada. Antes de llegar a la tercera tutoría cinco de los cuatro grupos -Jane Goodall, El cuerpo humano, Rayos y Truenos, Agua y Crecimiento de Ios árboles, pidieron una nueva tutoría para reducir incertidumbres y dudas que existían entre los miembros del grupo y que dificultaban la correcta orientación del cuento.

En la tercera tutoría, se debía presentar el esbozo del cuento, casi de forma definitiva, donde únicamente quedaran pequeños detalles por terminar. En esta tutoría ya se debía contar a la 
vez que se enseñaba el cuento, y de esta manera poder determinar aquellos detallas a mejorar e, incluso, la adaptación del lenguaje de los conceptos científicos a la etapa de educación infantil. Cierto es que pocos grupos tenían casi finalizado el cuento y que la mayoría presentaron algo más elaborado lo que habían mostrado en la anterior tutoría, aunque en este momento ya tenían la historia construida. Cabe destacar que esta tercera tutoría se celebró antes de Semana Santa lo que facilitó que durante esos días no lectivos el alumnado pudiera terminar el cuento. A la vuelta todos los grupos pidieron una tutoría con la cual afianzar el cuento definitivo, en ella se volvió a representar el cuento.

Terminó la experiencia en las aulas de la etapa de educación infantil. Fue el centro CEIP Salgado Torres, la dirección del centro y las tutoras de los cursos de cuarto y quinto de infantil, quien nos abrieron sus puertas para rematar la práctica. Para la asistencia al centro se tuvo en cuenta no provocar disrupciones con el resto de materias que cursa el alumnado en la facultad. Por eso se acudió a las aulas de infantil en el horario propio de la materia, distribuyendo al alumnado entre esos dos días: lunes con nueve grupos porque el tiempo era mayor de dos horas que permitía llegar a la siguiente clase en la facultad, y el miércoles con el resto de grupos, cinco, con un tiempo menor de $12 \mathrm{~h} 30$ a 13h30. Cada grupo estuvo en un aula de cuarto y de quinto para leer, explicar, representar y mostrar el cuento. Al finalizar la lectura, cada grupo debía de entregar el cuento definitivo. Se muestra a continuación algunos ejemplos en la imagen 1. El alumnado de educación infantil pidió quedarse algunos de los cuentos como: "Agua”, "Crecimiento de los árboles” y “¿Por qué llueve?”. 


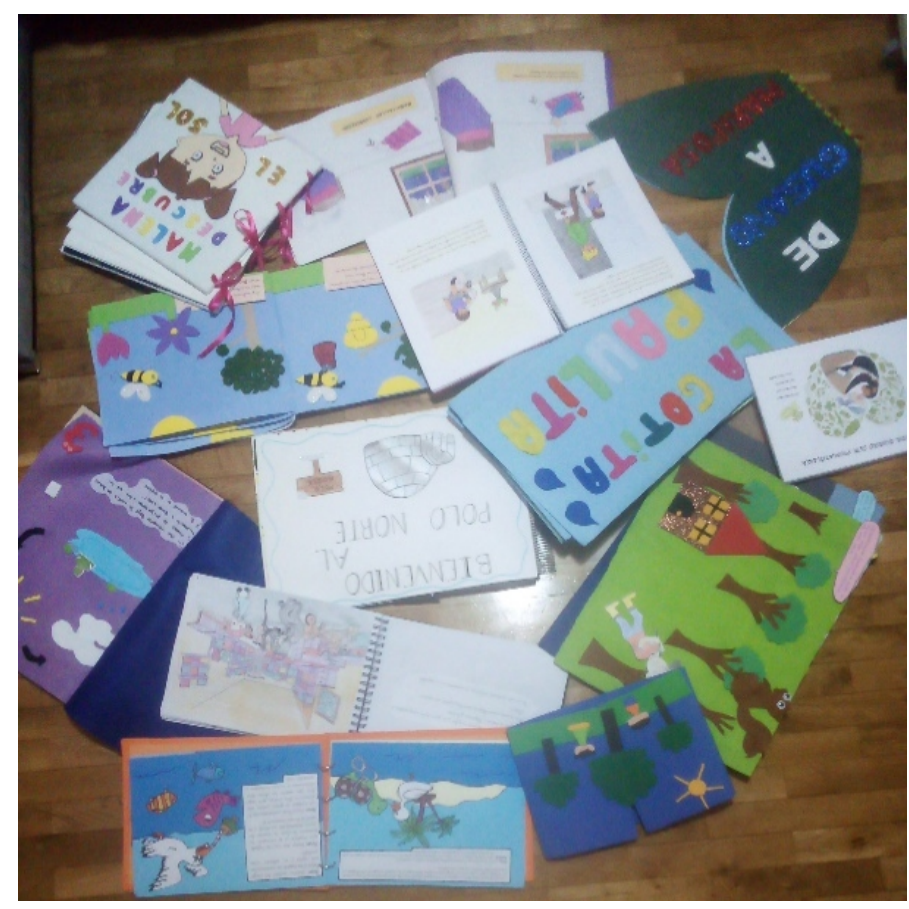

Figura 1. Portadas y páginas de los cuentos. (elaboración propia)

\section{RESULTADOS}

Al final de la actividad se pasó un cuestionario. En él se preguntaba por datos académicos y personales que nos sitúan el perfil del alumnado del grado y, además, averiguar los siguientes grados: aceptación de la actividad, interés, logro de expectativas, parte más difícil en la realización de la actividad y parte más fácil. Por último, se pidieron unas propuestas de mejora de la actividad. Excepto esta última, el resto de grados estaban construidos en una escala descriptiva numérica de 1 a 5 donde 1 era mínimo y 5 , máximo. En los ítems que se preguntó por la parte más fácil y la más difícil al elaborar el cuento se encontraron las siguientes entradas: búsqueda de información; dibujar; sintetizar y adaptar la información; maquetar; y, trabajo en equipo. Debían numerar en orden creciente cada una de las entradas en función de aquello que se pedía. 
El perfil del estudiante de la materia de Ensino das Ciencias da Natureza de segundo del grado de educación infantil es: una alumna proveniente de bachiller con 5 años o menos alejada de materias relacionadas con las ciencias de la naturaleza, una edad entre 19 y 31 años siendo el $72.9 \%$ entre 19 a 21 años. Resumimos en las tablas 1 a 3 siguientes estos datos.

\begin{tabular}{llll}
\hline Estudios & Porcentaje & Estudios & Porcentaje \\
\hline Bachillerato & $64.4 \%$ & Otros C. F. & $3.4 \%$ \\
C.F. Ed. Infantil & $27.1 \%$ & $\begin{array}{l}\text { Estudios } \\
\text { universitarios }\end{array}$ & $1.7 \%$
\end{tabular}

Tabla 1. Porcentaje procedencia estudios (elaboración propia).

\begin{tabular}{llll}
\hline Edad & Porcentaje & Edad & Porcentaje \\
\hline 19 & $40.7 \%$ & 25 & $3.4 \%$ \\
20 & $20.3 \%$ & 26 & 0 \\
21 & $11.9 \%$ & 27 & $1.7 \%$ \\
22 & $8.5 \%$ & 28 & $0 \%$ \\
23 & $8.5 \%$ & 29 & $1.7 \%$ \\
24 & $1.7 \%$ & 30 & 0 \\
& & 31 & $1.7 \%$ \\
\hline
\end{tabular}

Tabla 2. Porcentaje edad alumnado (elaboración propia).

\begin{tabular}{llll}
\hline Años sin CCNN & Porcentaje & Años sin CCNN & Porcentaje \\
\hline 1 & $5.1 \%$ & 7 & $3.4 \%$ \\
2 & $16.9 \%$ & 8 & 5.1 \\
3 & $11.9 \%$ & 9 & $3.4 \%$ \\
4 & $20.3 \%$ & 10 & $3.4 \%$ \\
5 & $22.0 \%$ & 11 & $0 \%$ \\
6 & $6.8 \%$ & 12 & $1.7 \%$
\end{tabular}

Tabla 3. Porcentaje años sin ciencias de la naturaleza (elaboración propia). 
Respecto a los grados, los porcentajes expresados por el alumnado son los que se reflejan en la tabla 4. En ella, se puede constar valores muy elevados en los tres grados, siendo en los tres el valor máximo, 5, el de mayor porcentaje presentado.

\begin{tabular}{clll}
\hline Valor & \multicolumn{1}{c}{$\begin{array}{c}\text { Gr. } \\
\text { aceptación }\end{array}$} & $\begin{array}{c}\text { Gr. } \\
\text { interés }\end{array}$ & $\begin{array}{c}\text { Logro } \\
\text { expectativas }\end{array}$ \\
\hline 1 & 0 & 0 & 0 \\
2 & 0 & 0 & 0 \\
3 & $4.7 \%$ & 0 & $4.7 \%$ \\
4 & $37.2 \%$ & $37.2 \%$ & $44.2 \%$ \\
5 & $58.1 \%$ & $62.8 \%$ & $51.2 \%$ \\
\hline
\end{tabular}

Respecto a la parte más fácil y la más difícil del trabajo, se contesta por el trabajo en equipo con un $48.8 \%$ como la más fácil, y sintetizar y maquetar como lo menos fácil con un $9.3 \%$. Lo más difícil fue esta misma respuesta con un $65.1 \%$, y lo menos difícil fueron dibujar y el trabajo en equipo ambos con un $7.0 \%$. Podemos contrastar en la tabla 5 los valores dados por el alumnado.

\begin{tabular}{lll}
\hline Valor & $\begin{array}{c}\text { Parte más } \\
\text { difícil }\end{array}$ & $\begin{array}{c}\text { Parte más } \\
\text { fácil }\end{array}$ \\
\hline 1 Búsqueda & $11.6 \%$ & $13.9 \%$ \\
información & & $16.3 \%$ \\
\hline Dibujar & $7.0 \%$ & 9.3 \\
3 Sintetizar y & & \\
adaptar & $65.1 \%$ & $11.6 \%$ \\
información & & $48.8 \%$ \\
\hline $\begin{array}{l}\text { 5 Maquetar } \\
\text { 5Trabajo en }\end{array}$ & $9.3 \% \%$ & \\
equipo & $7.0 \%$ & \\
\hline
\end{tabular}

Tabla 5. Porcentajes partes del diseño del cuento (elaboración propia). 
Por último, se pidió en el cuestionario propuestas de mejora siendo el $53.5 \%$ de las respuestas obtenidas "nada" y "todo bien". La siguiente más contestada es aumentar el número de tutorías con el $11.6 \%$ de las respuestas, y le sigue más tiempo de trabajo en el aula con un $9.3 \%$ y realizarlo en otros formatos como por ejemplo obras teatrales con $7.0 \%$. Destacar algunas de las respuestas indicadas, hacen referencia a la evaluación de la actividad por el porcentaje existente en la guía docente, también respuestas como elección más libre de los temas y la devolución de los trabajos, todas ellas con un $4.7 \%$. Por último, un grupo de respuestas menos numerosos que destacan la pérdida de tiempo al ir a la escuela a contar los cuentos y otras respuestas que piden ir a más aulas ambas con un $2.3 \%$.

\section{CONCLUSIONES}

En definitiva, concluir que la actividad resultó interesante para el aprendizaje porque el concepto tratado se consiguió que lo asimilasen y entendiesen mucho mejor. Además, la aceptación de la actividad y el interés despertado en el alumnado fue muy elevada siendo la percepción de las alumnas de conseguir las expectativas que tenían depositadas en esta actividad, en este recurso metodológico para su futuro docente.

Es importante que a lo largo del periodo de acompañamiento en la elaboración de futuros cuentos haya mayor énfasis para reducir las dificultades en la transposición didáctica de los conocimientos científicos a un lenguaje adaptado al alumnado. Además, aunque la puerta estaba siempre abierta y la disposición era total a los mails pidiendo tutorías, haría falta elaborar más tutorías que facilitaran el acompañamiento del alumnado porque no solo piden más tutorías, sino que piden más horas en aula dedicadas a esta actividad.

Únicamente se ofreció una plataforma para el diseño y elaboración de historias y cuentos que permitía la redacción y el dibujo, pero ninguno de los grupos escogió esta modalidad para representarlo. Desde el punto de vista docente, cabría la posibilidad de ampliar la oferta de plataformas que facilitan la producción de cuentos e historias digitalmente, se debería realizar una búsqueda de esta posibilidad tecnológica. No se preguntó por los motivos que suscitaron 
la no elección de plataformas digitales. Nos hace pensar en dos grandes motivos: primero, la facilidad en la elaboración de forma digital; o bien, las dificultades en el uso de los recursos existentes en la plataforma propuesta "Storyjummper". Este segundo motivo tiene su razón de ser cuando el alumnado pide más plataformas, más posibilidades a escoger para realizarlo en modalidad digital.

Además, se hace necesario corregir el sistema de evaluación de la materia para contribuir a un mayor peso de esta actividad. El alumnado siente que han aprendido y que la actividad tendrá beneficios en su futuro profesional, pero no ven recompensado el esfuerzo realizado con los porcentajes, con el peso dentro de la evaluación de la materia que representa esta actividad. que les resulta gratificante e interesante para su aprendizaje y para su futuro profesional como maestras en esta etapa educativa.

\section{REFERENCIAS}

Ausubel, D. P., Novak, J. D., \& Hanesian, H. (1983). Psicología educativa. Un punto de vista cognoscitivo. México: Trías editorial.

Blanco, B. y Blanco, L. J. (2009). Cuentos de matemáticas como recurso en la enseñanza secundaria obligatoria. Innovación Educativa, 19k, 193-206.

Chevallard, Y. (1991). La transposition didactique: du savoir savant au savoir enseigné. Grenoble: La Pensée Sauvage.

Chi, M. T. H., \& Roscoe, R. D. (2002). The processes and challenges of conceptual change. En M. Limon y L. Mason (Ed.) Reconsidering conceptual change: Issues in theory and practice, 3-27. Dordrecht: Springer. https://doi.org/10.1007/0-306-47637-1_1

Dulsat, C. (2019). Microenseñanza en el laboratorio de ciencias para el alumnado del grado de educación infantil. Revista Científica, 36(3), 293-306. https://doi.org/10.14483/23448350.14769

Espinet, M. (1995). El papel de los cuentos como medio de aprendizaje de las ciencias en educación infantil. Aula de Innovación Educativa, 44, 59-64. 
Fernández, R., Harris, C., y Aguirre, C. (2014). Propuestas para el tratamiento de la Competencia Matemática y de Ciencias a través de la literatura infantil en Educación Infantil y Primaria. NúMEROS, Revista de Didáctica de las Matemáticas, 85, 25-39. http://www.sinewton.org/numeros

García, S., y Pérez, J. M. (2016). Enseñanza de las ciencias naturales en educación primaria a través de cuentos y preguntas mediadoras. RIDHyC, Enseñanza de las Ciencias Naturales, 3, 101-122.

García-Castejón, M. M. (2013). Una narrativa en la enseñanza de las ciencias de la naturaleza. Investigación en la Escuela, 79, 79-85. http://dx.doi.org/10.12795/IE.2013.i79.07

Guevara, E. (2016). El cuento como herramienta de intervención. Infancia, Educación y Aprendizaje (IEYA), 2(1), 188-189. https://revistainfanciaeducacionyaprendizaje.com

Martínez, N. (2011). El cuento como instrumento educativo. Innovación y experiencias educativas, 39, 1-8.

Mateos, A., Bejarano, M., y Moreno, D. (2015). Los cuentos y los juegos de simulación para trabajar la justicia social en el ámbito de las ciencias en las primeras edades. Revista Internacional de Educacion para la Justicia Socia, RIEJS, 3(1), 97-119.

Orellana, M., y Espinet, M. (2009). Los cuentos como una herramienta para la modelización compleja del entorno en la formación inicial de maestros de ciencias. Enseñanza de las Ciencias, número extra VIII Congreso Internacional sobre Investigación en Didáctica de las Ciencias, Barcelona, 2733-2737. http://ensciencias.uab.es/congreso09/numeroextra/art2733-2737.pdf

Pardial, R., y Sáenz-López, P. (2013). Los cuentos populares/tradicionales en educación infantil. Una propuesta a través del juego. E-motion. Revista de Educación, Motricidad e Investigación, 2, 32-47. http://www.uhu.es/publicaciones/ojs/index.php/e-moti-on/index

Pérez, M. L. (2016). ¿Cómo trabajar las ciencias sociales en la educación infantil? Publicaciones Didácticas, 78, 56-59.

Pérez, D., Pérez, A. I., y Sánchez, R. (2013). El cuento como recurso educativo. 3c Empresa: investigación y pensamiento crítico, 2(4), 1-29. http://ojs.3ciencias.com/index.php/3cempresa/article/view/156 